\section{REVISTA BRASILEIRA DE QUALIDADE DE VIDA}

\title{
Prevalência de dor lombar e qualidade de vida no terceiro trimestre de gestação
}

\author{
Prevalence of low back pain and quality of life in the third trimester of \\ pregnancy
}

\author{
Denise Nakawatase \\ Faculdade de Ciências Médicas da Santa Casa de São Paulo - FCMSCSP - São Paulo - SP - Brasil \\ denisenakawatase@yahoo.com.br \\ Vera Lúcia dos Santos Alves \\ Faculdade de Ciências Médicas da Santa Casa de São Paulo - FCMSCSP - São Paulo - SP - Brasil \\ fisioterapiasc@uol.com.br \\ Eduardo Filoni \\ Universidade de Mogi das Cruzes - UMC - São Paulo - SP - Brasil \\ edufiloni@hotmail.com
}

\section{RESUMO}

OBJETIVO: Correlacionar a dor lombar no terceiro trimestre gestacional com a qualidade de vida (QV).

MÉTODOS: A amostra foi composta por 120 mulheres no terceiro trimestre de gestação, sendo 40 gestantes no $7^{\circ}$ mês $\left(28^{\mathrm{a}}\right.$ semana à $31^{\mathrm{a}}$ semana), 40 gestantes no $8^{\circ}$ mês $\left(32^{\mathrm{a}}\right.$ semana à $35^{\mathrm{a}}$ semana) e 40 gestantes no $9^{\circ}$ mês ( $36^{\mathrm{a}}$ semana à $39^{\mathrm{a}}$ semana). Trata-se de um estudo descritivo de paradigma quantitativo e corte transversal. Foram utilizados como instrumentos para a coleta de dados: a ficha de avaliação, a Escala Analógica de Dor e Incapacidade Funcional (EADIF) e o questionário WHOQOL-bref. Para a avaliação dos dados foi utilizada análise estatística descritiva (média, desvio padrão, mínimo e máximo). O teste de correlação de Spearman foi aplicado para determinar a confiabilidade das medidas.

RESULTADOS: Das gestantes analisadas, 80,83\% apresentam lombalgia. Quanto à intensidade da dor, aumentou com o tempo de gestação. $\mathrm{Na} 29^{\mathrm{a}}$ semana, houve um pico de dor e entre a $35^{\mathrm{a}}$ à $37^{\mathrm{a}}$ semanas também, apresentando uma escala entre 'pouca dor' e 'dor razoável'. Observou-se que o domínio físico é o que melhor se correlaciona, tanto com o escore total do Whooqol-bref, quanto no da EADIF. Os índices de QV demonstraram que as gestantes apresentaram um intervalo entre 'nem satisfatório, nem insatisfatório' e 'satisfatório'.

CONCLUSÕES: As modificações físicas e emocionais que acontecem durante a gestação nem sempre constituem os fatores intervenientes na QV das gestantes.

PALAVRAS-CHAVE: Gestação. Dor lombar. Qualidade de vida. 


\section{ABSTRACT}

OBJECTIVE: To assess the prevalence of low back pain and quality of life in the third trimester. METHODS: Were evaluated for sample 120 women in the third trimester of pregnancy, 40 pregnant women at 7 months ( 28 weeks to 31 weeks), 40 women in 8 months (32 weeks to 35 weeks), and 40 women in the 9th month (36th week of 39th week). This is a descriptive study of the quantitative paradigm and cross section. Was used as instruments: evaluation form, questionnaire EADIF and also the quality of life questionnaire WHOQOL-bref. For the evaluation of the data was analyzed using descriptive statistics (average, standard deviation, minimum and maximum). The Spearman correlation test was applied to determine the reliability of the measurements.

RESULTS: Among 100\% of pregnant women analyzed, $80.83 \%$ had low back pain. Regarding pain intensity increased with gestational age. At 29 weeks, there was a peak of pain between the 35th and the 37th week also showing a scale between "little pains" and "pain reasonable." It was observed that the physical domain is the best correlates both with the total score of the WHOQOL, as in the EADIF.

CONCLUSIONS: Therefore, one can conclude that the physical and emotional changes that occur during pregnancy are not always intervening factors on QL of pregnant women

KEYWORDS: Pregnancy. Back pain. Quality of life.

\section{Introdução}

Durante o período gestacional observam-se intensas alterações fisiológicas, funcionais e emocionais na mulher. O sistema músculo esquelético modifica-se e passa a adaptar a postura estática ou dinâmica de forma compensatória, produzindo queixas de dor e desconforto, além de causar limitações durante a execução de suas atividades de vida diária (MARTINS; SILVA, 2005). Tal sintoma é importante devido aos seus prejuízos, podendo causar transtornos sociais, tais como: carregar objetos, limpar a casa, sentar e caminhar, além de poder acarretar absenteísmo e distúrbios do sono. Desta forma, pode causar incapacidades, interferindo na qualidade de vida $(\mathrm{QV})$ das gestantes (ASSIS; TIBÚRCIO, 2004).

De acordo com Rezende e Montenegro (2006), as alterações fisiológicas acontecem desde a postura corporal da gestante aos outros sistemas de seu organismo, como respiratório, cardiovascular, gastrointestinais, endócrino e urinário.

A lombalgia na gestação vem sendo uma das principais queixas, sendo considerada como uma ocorrência normal e, até mesmo, esperada. Este sintoma pode incidir na região lombar irradiando-se ou não para os membros inferiores (FERREIRA; PITANGUI; NAKANO, 2006; SABINO; GRAUER, 2008). Além disso, os desconfortos da lombalgia podem permanecer por um período de até três anos após o parto e ela é uma das principais causas de absenteísmo no trabalho entre as gestantes (COLLITON, 1996).

De acordo com Aguiar, Silva e Pereira (2007), a lombalgia é frequente na gestação, independente da idade gestacional (IG). Apesar de comum, deve ser tratada e não simplesmente encarada como consequência normal da gravidez, pois gera grande repercussão à saúde da mulher no período gravídico-puerperal.

As etiologias mais prováveis da modificação na postura da gestante estão ligadas a uma relevante porcentagem da massa adquirida e ganho na região abdominal anterior à linha da gravidade, em decorrência do aumento do útero e das mamas, provocando um deslocamento anterior do centro de gravidade que resulta em uma maior instabilidade, ocasionando, também, maior estresse na musculatura abdominal e na região lombar (RIBAS; GUIRRO, 2007; SABINO; GRAUER, 2008).

O objetivo do presente estudo é correlacionar a dor lombar no terceiro trimestre gestacional com a QV. 


\section{Material e métodos}

Trata-se de um estudo transversal, analítico e descritivo. Para composição da amostra foi utilizado o método não probabilístico intencional. O projeto foi previamente aprovado pelo Comitê de Ética e Pesquisa de Seres Humanos da Santa Casa de São Paulo. A amostra foi constituída de 120 mulheres no terceiro trimestre de gestação, sem síndromes compressivas e que não tiveram gravidez gemelares. Todas as gestantes assinaram o termo de consentimento, foram abordadas e questionadas sobre a IG, se sentiam dor lombar ou não e se tinham disponibilidade para a realização da entrevista.

A coleta de dados utilizou três instrumentos distintos.

O primeiro foi uma ficha de avaliação composta por dados pessoais e anamnese específica.

A coleta de dados sobre possíveis queixas de dor e desconforto das gestantes foram feitas por meio do questionário Escala Analógica de Dor e Incapacidade Funcional (EADIF). A EADIF tem como objetivo mensurar a incapacidade funcional decorrente de dor lombar, através de 27 questões que avaliam a intensidade da dor em uma escala de 0 a 4 ( 0 nenhuma dor; 1 pouca dor; 2 dor razoável; 3 dor muito forte, mas suportável; 4 dor insuportável). O resultado final é obtido pelo somatório das pontuações de cada item totalizando o máximo de 108 pontos (MONNERAT; PEREIRA, 2009).

O questionário de QV da Organização Mundial da Saúde (OMS), o WHOQOL-bref (World Health Organization Quality of Life Assessment - short version), constituído por 26 questões divididas em 4 domínios: físico (7 questões), psicológico (6 questões), relacões sociais (4 questões) e meio ambiente (9 questões) foi o terceiro instrumento utilizado (FLECK et al., 1999; KLUTHCOVSKY; KLUTHCOVSKY, 2009; OLIVECRONA et al., 2009). A pontuação compreende a soma dos valores de cada questão, que estão organizados numa escala do tipo Likert, que vai de 1 a 5, sendo que o significado depende do domínio avaliado. Quanto mais alto o valor, quando convertidas as questões invertidas, maior a QV (TIMOSSI et al., 2009).

Para a classificação dos resultados foi adotada uma escala centesimal de 0 a 100 , onde 0 valor 50 caracteriza o ponto central e os valores acima e abaixo do ponto central representam respectivamente elevados níveis de satisfação ou insatisfação em relação à QV (Tabela 1).

Tabela 1 - Escala de níveis de satisfação

\begin{tabular}{lccccc}
\hline \multicolumn{1}{c}{ Escala } & $\mathbf{0 \%}$ âncora & $\mathbf{2 5 \%}$ & $\mathbf{5 0 \%}$ & $\mathbf{7 5 \%}$ & $\mathbf{1 0 0 \%}$ âncora \\
\hline Avaliação do nível de satisfação & Muito insatisfeito & Insatisfeito \\
Grau numérico & 1 & 2 & $\begin{array}{c}\text { Nem satisfeito } \\
\text { nem insatisfeito }\end{array}$ & Satisfeito Muito satisfeito \\
\hline
\end{tabular}

Fonte: Adaptado de Fleck et al. (1999).

Para a avaliação dos dados foi utilizada a ferramenta desenvolvida por Pedroso et al. (2010), a partir do software Microsoft Excel, que permite o cálculo dos escores e da estatística descritiva do instrumento WHOQOL-bref.

Foi realizado levantamento da análise estatística descritiva (média, desvio padrão, mínimo e máximo) para a EADIF.

O teste de correlação de Spearman foi aplicado para determinar a confiabilidade das medidas. Para indicar a significância estatística foram considerados os testes $\mathrm{T}$ de Student $\mathrm{e}$ Wilcoxon. 


\section{Resultados}

\subsection{Caracterização da Amostra}

Neste estudo, a idade média das gestantes foi de 29,10 $( \pm 6,52)$ anos, peso 73,98 $( \pm 12,54)$, altura 1,62 ( \pm 0,06), com Índice de Massa Corporal (IMC) de 28,08 $( \pm 4,44 \mathrm{~kg} / \mathrm{m} 2)$ e IG média de 33,14 ( $\pm 3,42$ semanas).

Entre as 120 gestantes entrevistadas, $53(44,16 \%)$ eram primigestas e $67(55,84 \%)$ multigestas, independentemente de terem levado a gestação até o fim. física:

A Tabela 2 apresenta as variáveis avaliadas no estudo relacionadas com hábitos e atividade

Tabela 2 - Distribuição dos hábitos e atividade física

\begin{tabular}{lccc}
\hline \multicolumn{1}{c}{ Variáveis } & $\begin{array}{c}\text { Sim } \\
\text { n gestantes (\%) }\end{array}$ & $\begin{array}{c}\text { Não } \\
\text { n gestantes (\%) }\end{array}$ & $\begin{array}{c}\text { Total } \\
\text { n gestantes (\%) }\end{array}$ \\
\hline Tabagismo & $03(2,50 \%)$ & $117(97,50 \%)$ & $100(\%)$ \\
Etilismo & $01(0,83 \%)$ & $119(99,17 \%)$ & $100(\%)$ \\
Atividade Física & $09(7,50 \%)$ & $111(92,50 \%)$ & $100(\%)$ \\
\hline
\end{tabular}

Fonte: Autoria própria (2015).

\subsection{Escores das respostas das somas dos questionários de dor lombar}

A Tabela 3 representa o somatório dos escores das somas e das médias das respostas dos questionários. Através da soma da avaliação dos questionários realizou-se a análise descritiva:

Tabela 3 - Resultados descritivos da soma e da média dos questionários

\begin{tabular}{cccccc} 
& Média & Desvio Padrão & Mínimo & Máximo & Valor p \\
\hline Soma da avaliação & 35,78 & 20,98 & 0 & 92,00 & $\mathrm{p}<0,01$ \\
Média da avaliação & 1,33 & 1,26 & 0 & 3,41 & $\mathrm{p}<0,01$ \\
\hline \multicolumn{4}{c}{ Obs.: através do teste T de Student e Wilcoxon. } \\
Fonte: Autoria própria (2015).
\end{tabular}

\subsection{Dados da lombalgia}

Observou-se na Tabela 4 que, dentre as 120 gestantes analisadas, $97(80,83 \%)$ apresentavam lombalgia e $23(19,17 \%)$ não apresentam lombalgia. 
Tabela 4 - Distribuição de dor lombar por mês gestacional $(n=120)$

\begin{tabular}{lccc}
\hline \multicolumn{1}{c}{ Sim } & Não & Total \\
Mês Gestacional & n gestantes (\%) & n gestantes (\%) & n gestantes (\%) \\
\hline $7^{\circ}$ mês & $26(65,00 \%)$ & $14(35,00 \%)$ & $40(100 \%)$ \\
$8^{\circ}$ mês & $34(85,00 \%)$ & $06(15,00 \%)$ & $40(100 \%)$ \\
$9{ }^{\circ}$ mês & $37(92,50 \%)$ & $03(7,50 \%)$ & $40(100 \%)$ \\
TOTAL & $97(80,83 \%)$ & $23(19,17 \%)$ & $120(100 \%)$ \\
\hline
\end{tabular}

Fonte: Autoria própria (2015).

A Tabela 5, considerando o número de gestações das participantes da amostra, apresenta a distribuição de dor lombar.

Tabela 5 - Distribuição de dor lombar por gestações

\begin{tabular}{lccc}
\hline Gestações & $\begin{array}{c}\text { Sim } \\
\text { n gestantes (\%) }\end{array}$ & $\begin{array}{c}\text { Não } \\
\text { n gestantes (\%) }\end{array}$ & $\begin{array}{c}\text { Total } \\
\text { n gestantes (\%) }\end{array}$ \\
\hline Primigestas & $44(83,02 \%)$ & $09(16,98 \%)$ & $53(100 \%)$ \\
Multigestas & $53(79,10 \%)$ & $14(20,90 \%)$ & $67(100 \%)$ \\
TOTAL & $97(80,83 \%)$ & $23(19,17 \%)$ & $120(100 \%)$ \\
\hline
\end{tabular}

Fonte: Autoria própria (2015).

\subsection{Dados da Escala Analógica de Dor e Incapacidade Funcional relacionados aos meses e semanas gestacionais}

Gráfico 1 - Comparação da dor lombar em relação aos meses gestacionais

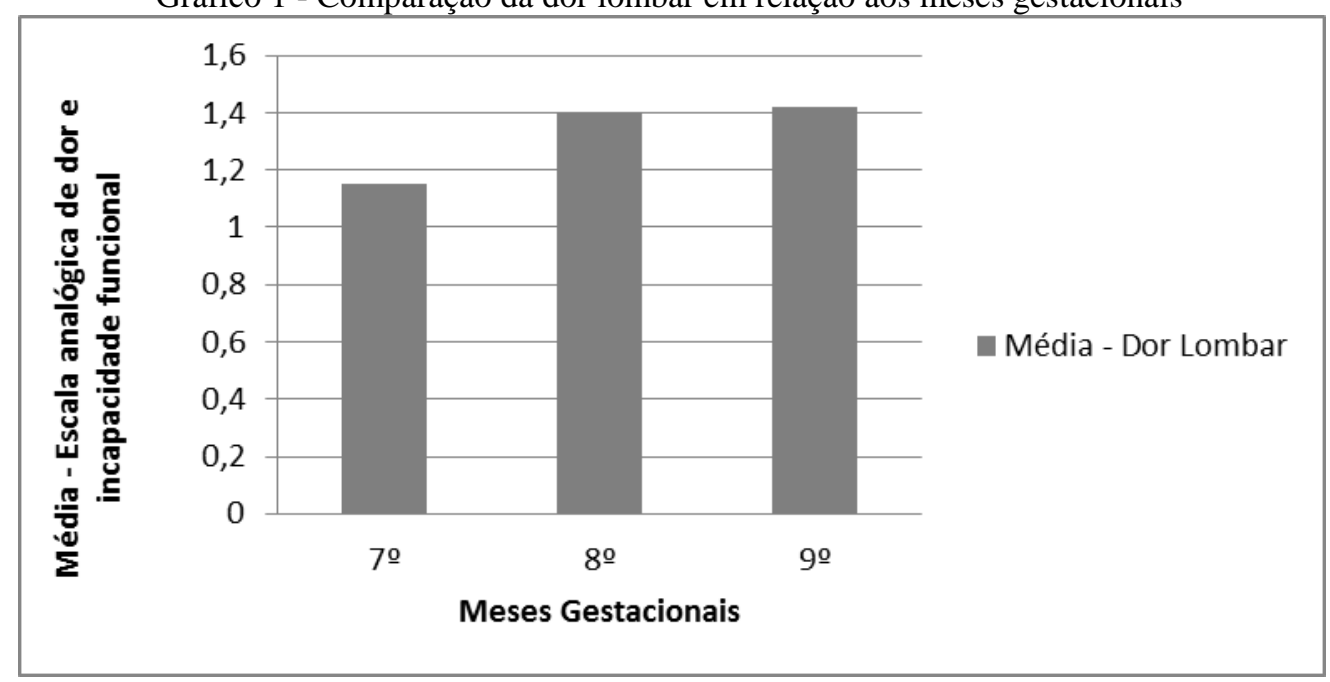

Fonte: Autoria própria (2015).

Em relação à lombalgia e à IG, pode-se observar que, com o avanço da IG, a dor lombar torna-se mais frequente e com maior intensidade, principalmente entre o $8^{\circ}$ mês e o $9^{\circ}$ mês $\left(35^{\mathrm{a}}\right.$ à 
$37^{\mathrm{a}}$ semanas), portanto dentro da EADIF estas gestantes apresentaram uma escala entre 'pouca dor' e 'dor razoável'. Mesmo havendo um pico de dor na 29a semana, a caracterização da dor na escala não foi alterada (Gráfico 2).

Gráfico 2 - Comparação da dor lombar em relação às semanas gestacionais

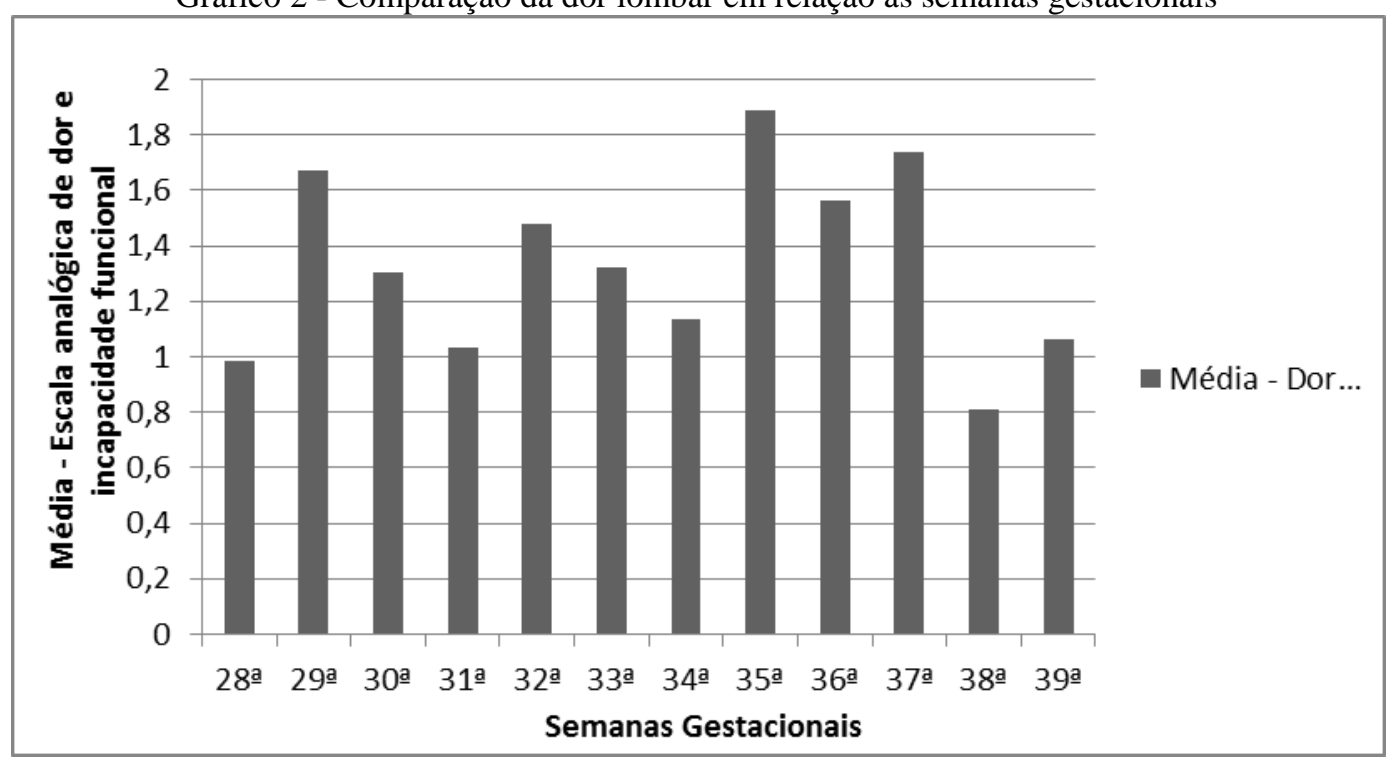

Fonte: Autoria própria (2015).

\subsection{Questionários de qualidade de vida quanto aos domínios}

A Tabela 6 apresenta os resultados das análises descritivas dos domínios (físico, psicológico, relações sociais e meio ambiente) por meio da avaliação do WHOQOL-bref:

Tabela 6 - Resultados descritivos gerais dos questionários de qualidade de vida

\begin{tabular}{lccccc}
\hline \multicolumn{1}{c}{ Domínio } & Média & Desvio Padrão & Coeficiente de variação & Valor mínimo & Valor máximo \\
\hline Físico & 12,79 & 2,58 & 20,17 & 7,43 & 17,71 \\
Psicológico & 15,21 & 1,94 & 12,73 & 8,67 & 20,00 \\
Relações Sociais & 15,76 & 2,81 & 17,83 & 5,33 & 20,00 \\
Meio Ambiente & 13,72 & 1,99 & 14,52 & 8,00 & 18,50 \\
Autoavaliação da QV & 15,80 & 2,60 & 16,44 & 6,00 & 20,00 \\
TOTAL & 14,21 & 1,67 & 11,76 & 8,15 & 17,69 \\
\hline
\end{tabular}

Fonte: Autoria própria (2015).

O Gráfico 3 mostra os resultados nos domínios considerados pelo WHOQOL-bref: 
Gráfico 3 - Comparação dos domínios em relação à qualidade de vida

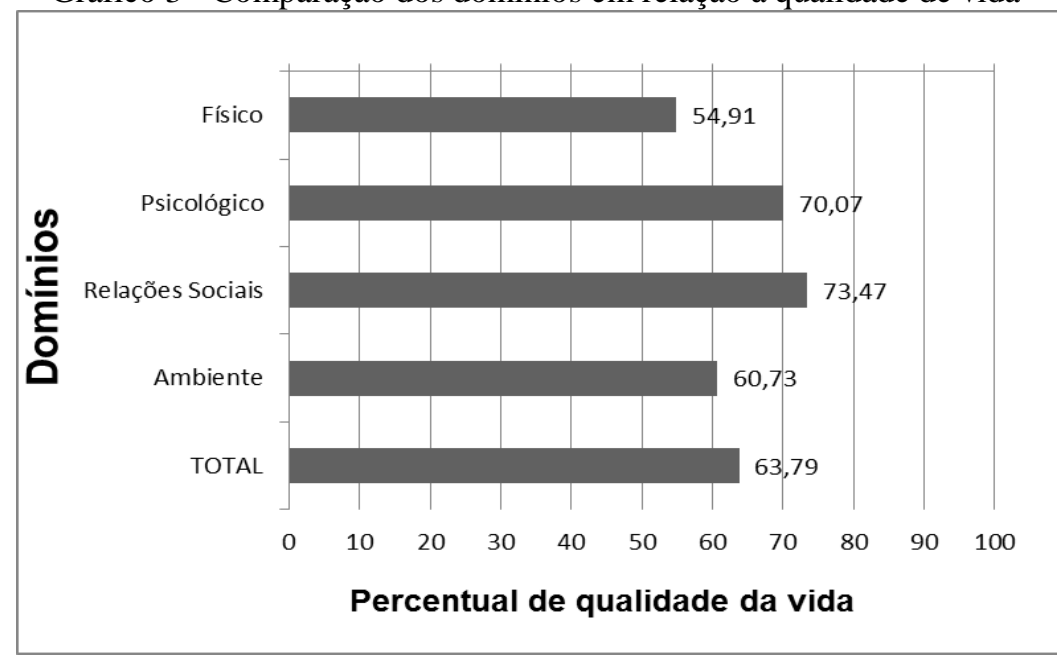

Fonte: Autoria própria (2015).

\subsection{Domínios da qualidade de vida relacionados aos meses gestacionais}

Na Tabela 7 são apresentados os resultados das análises descritivas dos domínios (físico, psicológico, relações sociais e meio ambiente) por meio da avaliação do WHOQOL-bref considerando três períodos distintos:

\begin{tabular}{lccc}
\multicolumn{4}{c}{ Tabela 7 - Distribuição dos domínios quanto ao mês gestacional } \\
\hline \multicolumn{1}{c}{ Domínio } & $\begin{array}{c}\mathbf{7}^{\mathbf{0}} \text { mês } \\
\text { Média (DP) }\end{array}$ & $\begin{array}{c}\mathbf{8}^{\mathbf{0}} \text { mês } \\
\text { Média (DP) }\end{array}$ & $\begin{array}{c}\mathbf{9}^{\mathbf{0}} \text { mês } \\
\text { Média (DP) }\end{array}$ \\
\hline Físico & $12,94(2,78)$ & $12,90(2,37)$ & $12,51(2,62)$ \\
Psicológico & $15,02(2,12)$ & $15,48(1,49)$ & $15,13(2,15)$ \\
Relações sociais & $15,90(2,37)$ & $15,47(3,09)$ & $15,90(2,96)$ \\
Meio ambiente & $13,69(1,79)$ & $14,00(1,81)$ & $13,46(2,34)$ \\
Autoavaliação da QV & $15,10(2,60)$ & $16,45(2,24)$ & $15,85(2,81)$ \\
& & & \\
TOTAL & $14,16(1,76)$ & $14,40(1,37)$ & $14,06(1,87)$
\end{tabular}

Fonte: Autoria própria (2015).

\subsection{Correlação dos dados gerais}

Para determinar o coeficiente de correlação entre variáveis foi utilizado como ferramenta estatística o coeficiente de correlação de Spearman (r). Para verificar a relação existente entre a QV e a EADIF foi realizada uma regressão linear observando o gráfico de dispersão e o valor do coeficiente de correlação para descobrir se existe uma relação forte e se é significativa através do valor de p. Foi considerado o diagrama de dispersão onde são plotados valores de x e y para a QV e a EADIF e o coeficiente de correlação linear. Com isto, pode ser observado através do Gráfico 4 de dispersão como os dados se comportaram: 
Gráfico 4 - Correlação entre qualidade de vida e escala analógica de dor e incapacidade funcional

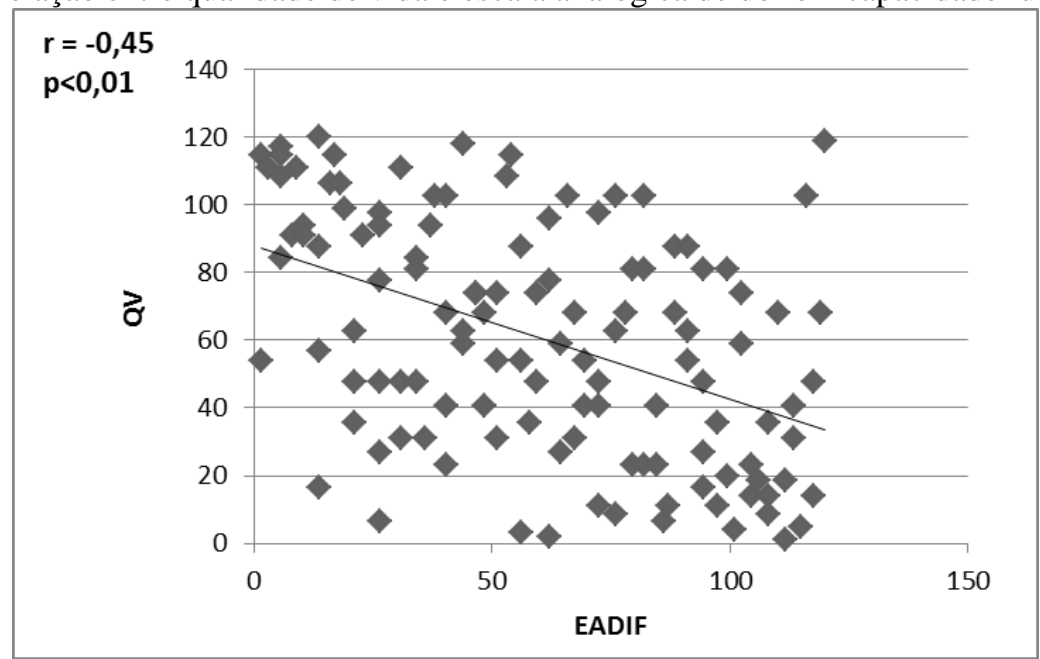

Fonte: Autoria própria (2015).

Utilizou-se o teste $\mathrm{T}$ de Student e Wilcoxon (teste não paramétrico) por se tratar de um procedimento extremamente útil. Todas as conclusões foram tomadas ao nível de significância de $99 \%(\mathrm{p}<0,01)$.

\subsection{Correlação dos dados da qualidade de vida por domínio versus da Escala Analógica de Dor e Incapacidade Funcional}

Diante dos dados obtidos pelo estudo, foram realizados cruzamentos entre determinantes (variáveis), obtendo-se valores com significância estatística. Do cruzamento entre as variáveis QV (domínio físico) versus EADIF (Gráfico 5), observa-se que $\mathrm{r}=-0,59, \mathrm{p}<0,01$, apresentam um coeficiente de correlação (Spearman) linear mais alto do que os demais domínios, no entanto, correlação negativa moderada significativa.

Os domínios: psicológico ( $\mathrm{r}=-0,27, \mathrm{p}<0,01)$, (relações sociais $(\mathrm{r}=-0,22, \mathrm{p}<0,05)$, meio ambiente $(\mathrm{r}=-0,12, \mathrm{p}<0,2)$ e autoavaliação $(\mathrm{r}=-0,19, \mathrm{p}<0,05)$ apresentaram coeficientes de correlação (Spearman) mais baixos. Todos os valores de $\mathrm{p}$ para os domínios citados foram analisados através do teste $\mathrm{T}$ de Student. Já no teste de Wilcoxon, todos os domínios obtiveram o valor de $\mathrm{p}<0,01$ conforme os Gráficos 5, 6, 7, 8 e 9 .

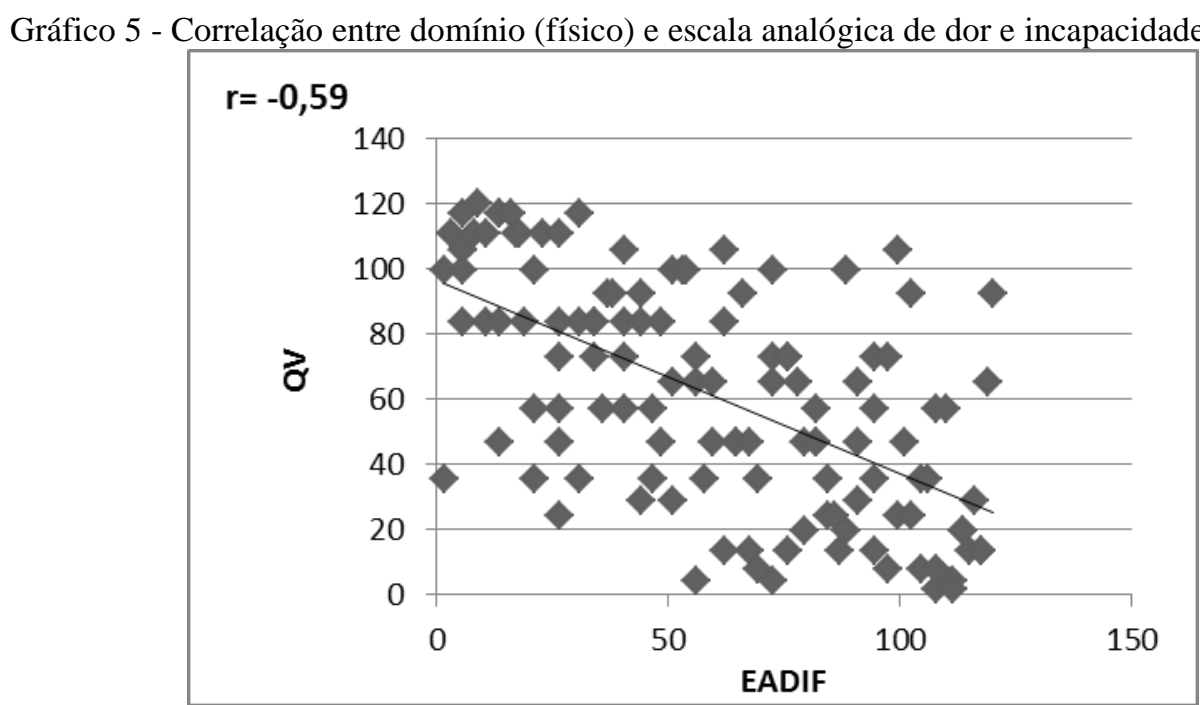

Fonte: Autoria própria (2015). 
Gráfico 6 - Correlação entre domínio (psicológico) e escala analógica de dor e incapacidade funcional

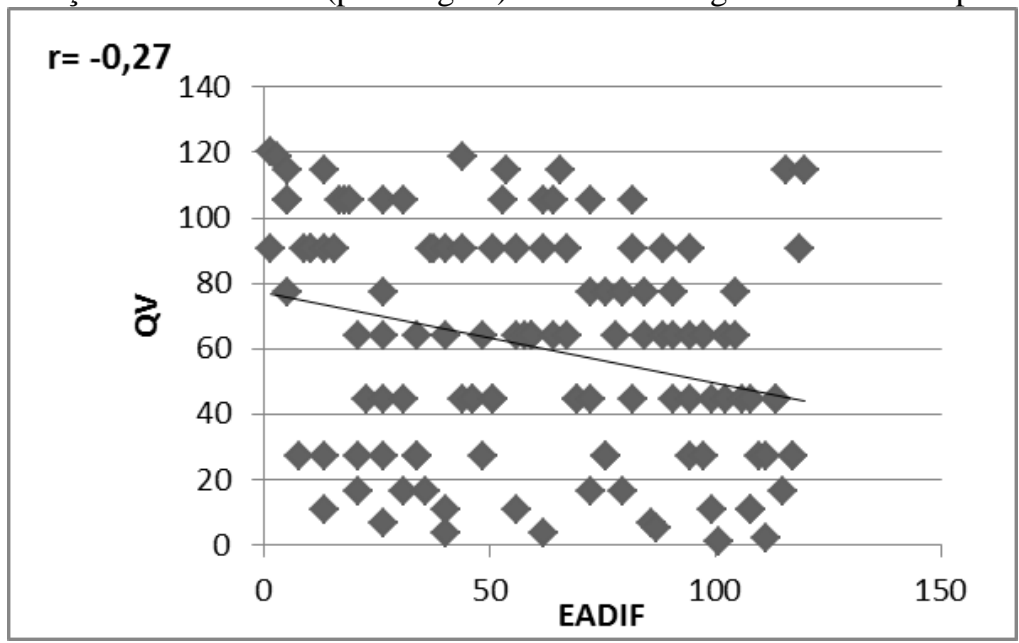

Fonte: Autoria própria (2015).

Gráfico 7 - Correlação entre domínio (relações sociais) e escala analógica de dor e incapacidade funcional

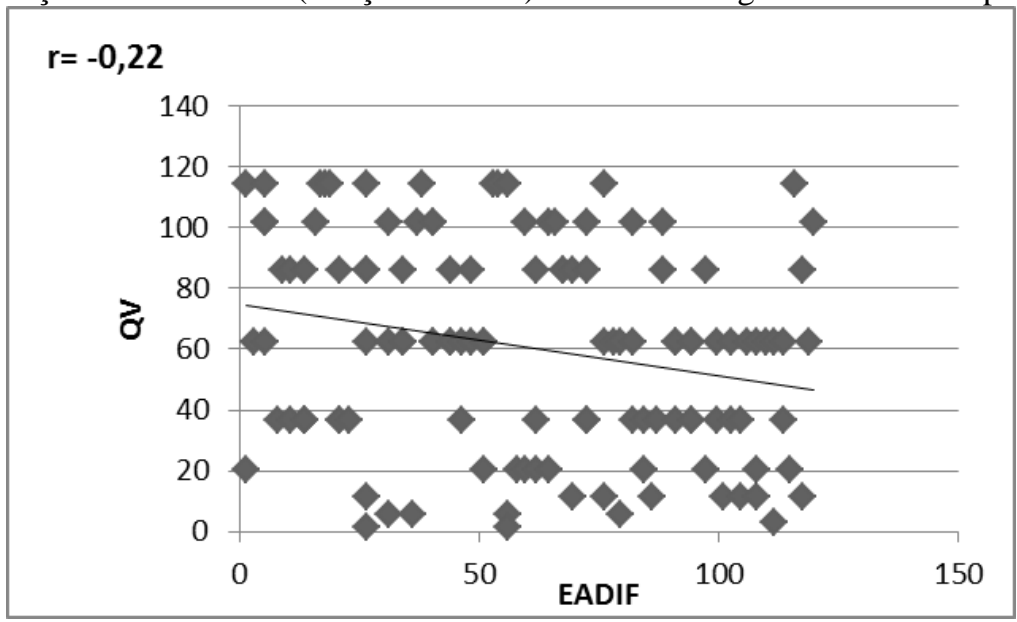

Fonte: Autoria própria (2015).

Gráfico 8 - Correlação entre domínio (meio ambiente) e escala analógica de dor e incapacidade funcional

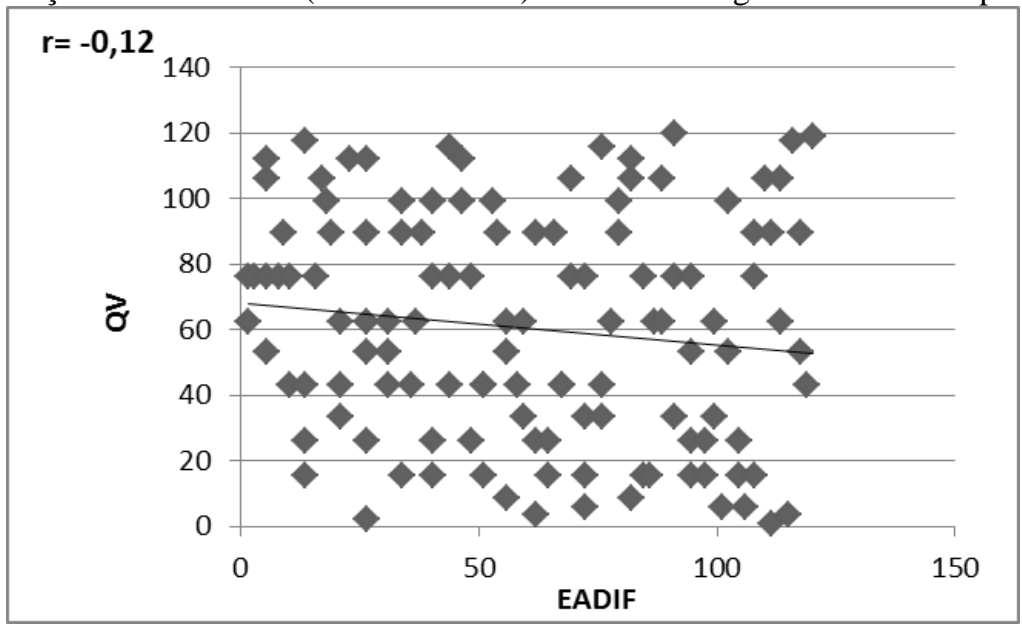

Fonte: Autoria própria (2015). 
Gráfico 9 - Correlação entre domínio (autoavaliação QV) e escala analógica de dor e incapacidade funcional

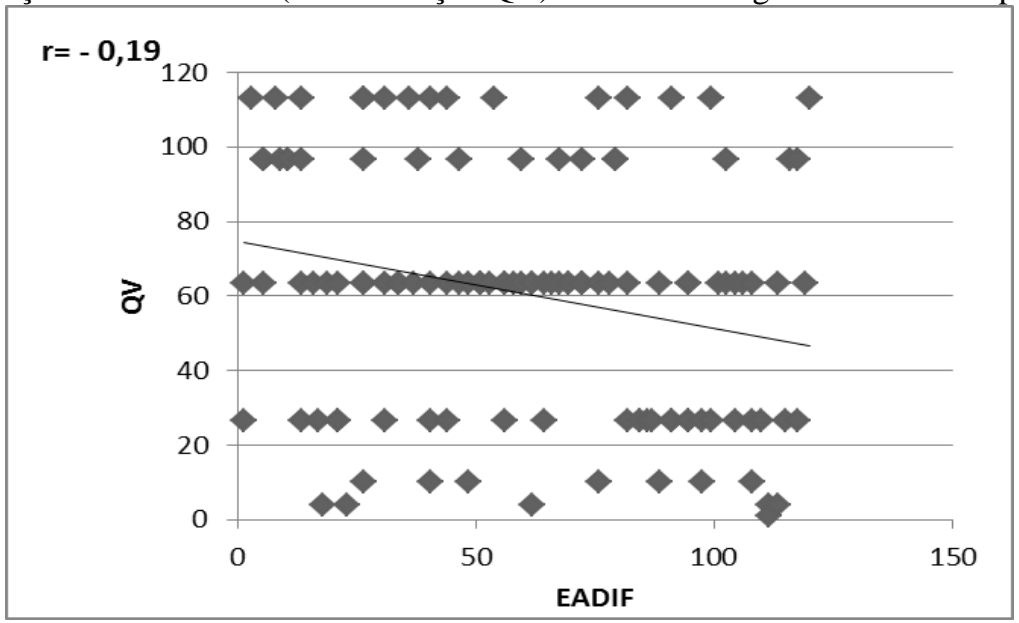

Fonte: Autoria própria (2015).

\section{Discussão}

O presente estudo constatou que a prevalência de lombalgia gestacional foi de $80,83 \%$, o que condiz com os dados da literatura, inclusive brasileira, que indicam taxas de 75 a $85 \%$ (KRISTIANSSON; SVÄRDSRDD; SCHOULTZ, 1996; KIHLSTRAND et al., 1999). Entre os fatores de risco associados ao desenvolvimento de dor lombar em gestantes estão a idade, a dor lombar prévia e o IMC (WANG et al., 2004; MOGREN; POHJANEN, 2005).

Atribui-se como uma das causas principais o hormônio relaxina, que torna as articulações mais instáveis, predispondo as estruturas ao estresse e à dor (FERREIRA; PITANGUI; NAKANO, 2006). A ocorrência da ativação constante dos músculos posteriores da coluna para compensar essa instabilidade também leva à fadiga muscular e à dor lombar (SIHVONEN et al., 1998). O menor desempenho do transverso do abdômen influencia a presença da lombalgia, já que esse músculo é participante da mecânica respiratória e responsável pela manutenção da postura corporal (NOVAES; SHIMO; LOPES, 2006).

Estudos demonstram que, quanto menor a idade, maior a chance de ter dor lombar na gestação (ÖSTGAARD; ANDERSSON, 1991; KRISTIANSSON; SVÄRDSRDD; SCHOULTZ, 1996; WANG et al., 2004; MARTINS; SILVA, 2005). Enquanto Mantle, Greenwood e Currey (1977) observaram que, com o aumento da idade, há maior prevalência de lombalgia. Orvieto et al. (1994) não encontraram correlação entre a idade materna e a lombalgia gestacional.

Em mais da metade da casuística, a intensidade da dor aumentou com o tempo de gestação, entre o $8^{\circ}$ mês e o $9^{\circ}$ mês ( $35^{\mathrm{a}}$ à $37^{\mathrm{a}}$ semanas), com escala entre 'pouca dor' e 'dor razoável'. Mesmo havendo um pico de dor no $7^{\circ}$ mês ( $29^{\mathrm{a}}$ semana), o que era esperado com o crescimento do útero e a sobrecarga na coluna lombar, a escala permaneceu entre 'pouca dor' e 'dor razoável' durante pico, o mesmo patamar dos meses seguintes. Embora que, no estudo de Wang et al. (2004), tenha se mostrado que a prevalência de lombalgia não foi afetada pela IG, Assis e Tibúrcio (2004) relataram que em $60 \%$ dos casos havia essa ocorrência e que a maior prevalência está no segundo trimestre. Kristiansson, Svärdsrdd e Schoultz (1996) mostraram que a incidência aumenta a partir da $24^{\mathrm{a}}$ semana de gestação.

Em um estudo realizado por Sihvonen et al. (1998), no qual foi utilizada a Escala Visual Analógica (VAS) para avaliação da intensidade da lombalgia na gestação, constatou-se que o quadro se apresentou maior no $1^{\circ}$ e no $3^{\circ}$ trimestre gestacional. Segundo Orvieto et al. (1994), a frequência foi maior nas gestantes que estavam no último mês. Para Carvalho e Caromano (2001), não há relação significativa entre os trimestres gestacionais. Acredita-se que a prevalência de sintomas dolorosos na região lombar continue presente no período gestacional e, dependendo do grau de dor, podem perdurar alguns anos após o parto, caso seja negligenciado seu tratamento (BATISTA et al., 2003). 
Dentre as gestantes entrevistadas, constatou-se que $44(83,02 \%)$ das primigestas e 53 $(79,10 \%)$ das multigestas (independentemente de terem levado a gestação até o fim) apresentam dor lombar. Segundo Östgaard (1996), Bouhassoun, Catanzariti e Thévenon (1998) e Galão, Zardo e Paula (1995), o número de gestações também constitui fator de risco para o aparecimento da lombalgia gestacional, o que diverge de outros estudos, como os de Fast et al. (1987) e Melzack e Belanger (1989), que não encontraram a mesma correlação. No entanto, isso não significa que as primigestas tenham menor chance de desenvolverem a dor lombar (MELHADO; SOLER, 2004).

Das gestantes avaliadas, $92,5 \%$ não praticam nenhum tipo de atividade física, o que também pode ter contribuído para a alta prevalência de lombalgia gestacional. Rodrigues et al. (2011), ao analisar 66 gestantes, com o objetivo de avaliar os fatores psicossociais de gestantes com queixas de lombalgias associada à falta de atividade física antes da gravidez, constataram existir relação entre dor e inatividade física. Adicionalmente, verificaram que mulheres praticantes de atividade física antes da gravidez foram menos acometidas por dores lombares.

O WHOQOL-bref versus a EADIF apresentaram coeficientes de correlação negativa moderada significativa, com todos os domínios do instrumento de QV. Observa-se que o domínio físico é o que melhor se correlaciona, tanto com o escore total do Whoqol-bref, quanto da EADIF. Todos os outros domínios não apresentaram resultados que indiquem alguma possível influência da presença de dor lombar na QV. Quanto à relação da QV e da EADIF, os domínios: psicológico, relações sociais, meio ambiente e autoavaliação da QV, não apresentaram uma correlação linear significativa em seus resultados. Hueston e Kasik-Miller (1998) notaram que as pontuações das medidas físicas de saúde se alteraram durante a gestação. No terceiro trimestre o peso do feto e do líquido amniótico, associado à fadiga, à redução da mobilidade e à sonolência podem ter levado à redução do domínio físico da QV quando comparado ao primeiro trimestre (JENSEN; DOUCET; TREITZ, 1996; SCHORR et al., 1998; CHOU; KUO; WANG, 2008; VAN DE POL et al., 2006; BORODULIN et al., 2010).

Rodrigues et al. (2011), analisando os fatores psicossociais, observaram que as gestantes que relataram presença da dor lombar tinham dificuldades em seus relacionamentos interpessoais. Nessa perspectiva, o estudo indica que o fator mais fortemente associado à alta taxa de relato de lombalgia é a qualidade deficiente das relações sociais (VALAT, 2005). Nessa mesma direção, Pimenta (1999) evidencia que o comprometimento no relacionamento social, profissional e afetivo agrava-se de acordo com a intensidade da dor. Quando os domínios foram avaliados separadamente, apresentaram-se com melhores escores os domínios: psicológico, relações sociais e autoavaliação da QV e, analisando a QV quanto ao mês gestacional, os domínios que apresentaram os melhores escores também foram: psicológico, relações sociais e autoavaliação da QV.

Diante dos resultados, observa-se que o valor total dos domínios encontrados é de $63,79 \%$. Portanto, os melhores resultados estão entre o domínio psicológico $(70,07 \%)$ e relações sociais (73,43\%). No estudo de Anthony e Scialli (1999), em pacientes com o estado de humor alterado, foi observado que a percepção de dor torna-se mais comum. Um dos fatores psicológicos que pode desencadear problema durante a gestação, parto e puerpério, bem como para o concepto, é o aumento do nível de ansiedade; quando reduzido esse nível, os efeitos das dores são minimizados (ARAÚJO et al., 2008). Pimenta (1999) afirma que todas as experiências humanas, inclusive as que causam dores crônicas, envolvem componentes físicos e emocionais (depressão e ansiedade), no entanto, o humor pode ficar comprometido interferindo na interpretação e no relato da dor.

\section{Considerações finais}

Evidenciou-se grande prevalência de dor lombar em contraste com QV (correlação moderada negativa significativa), o que sugere que a dor lombar apresenta etiologia multifatorial, fazendo com que as gestantes não tenham um período gestacional ideal. As modificações físicas e emocionais que acontecem durante a gestação nem sempre constituem os fatores intervenientes na QV das gestantes. 


\section{Referências}

AGUIAR, E. O. G; SILVA, M. A. G; PEREIRA, J. S. Frequência de dor lombar em grávidas e relação com a idade gestacional. Fisioterapia Brasil, Rio de Janeiro, v. 8, n. 1, p. 31-35, jan./fev. 2007.

ANTHONY, R.; SCIALLI M. D. Evaluating chronic pelvic pain. A consensus recommendation. The Journal of Reproductive Medicine, v. 44, n. 11, p. 945-952, 1999.

ARAÚJO, D. M. R.; PACHECO, A. H. de R. N.; PIMENTA, A. M; KAC, G. Prevalência e fatores associados a sintomas de ansiedade em uma coorte de gestantes atendidas em um centro de saúde do município do Rio de Janeiro. Revista Brasileira de Saúde Materno Infantil, v. 8, n. 3, p. 333340, 2008. Crossef

ASSIS, R. G; TIBÚRCIO, R. E. S. Prevalência e características da lombalgia na gestação: um estudo entre gestantes assistidas no programa de pré-natal na maternidade dona Íris em Goiânia. 2004. 31 f. Trabalho de Conclusão de Curso (Bacharelado em Fisioterapia) - Universidade Católica de Goiás, Goiânia, 2004.

BATISTA, D. C; CHIARA, V. L; GUGELMIN, A. S; MARTINS, P. D. Atividade física e gestação: saúde da gestante não atleta e crescimento fetal. Revista Brasileira de Saúde Materno Infantil, Rio de Janeiro, v. 3, n. 2, p. 151-158, abr./jun. 2003.

BORODUlin, K.; EVENSON, K. R.; MONDA, K.; WEN, F.; HERRING, A. H.; Dole, N. Physical activity and sleep among pregnant women. Paediatric and Perinatatal Epidemiology, v. 24, n. 1, p. 45-52, 2010. Crossef

BOUHASSOUN, N.; CATANZARITI, J. F; THÉVENON, A. Lombalgies et grossesse. Revue du Rhumatisme, v. 65, p. 59-62, 1998.

CARVALHO, Y. B. R; CAROMANO, F. A. Alterações morfofisiológicas relacionadas com lombalgia gestacional. Arquivos de Ciências da Saúde da Unipar, v. 5, n. 3, p. 267-272, 2001.

CHOU, F. H.; KUO, S. H.; WANG, R. H. A longitudinal study of nausea and vomiting, fatigue and perceived stress in, and social support for, pregnant women through the three trimesters. Kaohsiung Journal of Medical Science, v. 24, n. 6, p. 306-314, 2008. erossef

COLliton, J. Back pain and pregnancy: Active management strategies. Physician and Sports Medicine, v. 24, n. 1, p. 1-6, 1996.

FAST, A.; SHAPIRO, D.; DUCOMMUN, E. J.; FRIEDMANN, L. W.; BOUKLAS, T.; FLOMAN, Y. Lowback pain in pregnancy. Spine, v. 12, n. 4, p. 368-371, 1987. Crossef

FERREIRA, C. H. J.; PITANGUI, A. C. R.; NAKANO, M. A. S., Tratamento da lombalgia na gestação. Fisioterapia Brasil, v. 7, n. 2, p. 138-141, 2006.

FLECK, M. P. A; LEAL, O. F.; LOUZADA, S.; XAVIER, M.; CHACHAMOVICH, E.; VIEIRA, G.; SANTOS, L.; PINZON, V. Desenvolvimento da versão em português do instrumento de avaliação de qualidade de vida da OMS (WHOQOL-100). Revista Brasileira Psiquiatria, v. 21, n. 1, p. 19-28, 1999. rossef 
GALÃO, A. O.; ZARDO, E. A.; PAUlA, L. G. Lombalgia da gestação. Acta Médica, v. 5, p. 347353, 1995.

SABINO, J.; GRAUER, J. N. Pregnancy and low back pain. Current Reviews in Musculoskeletal Medicine, v. 1, n. 2, p. 137-141, June 2008. Crossef

HUESTON, W. J.; KASIK-MILLER, S. Changes in functional health status during normal pregnancy. Journal of Family Practice, v. 47, n. 3, p. 209-212, 1998.

JENSEN, R. K.; DOUCET, S.; TREITZ, T. Changes in segment mass distribution during pregnancy. Journal of Biomechanics, v. 29, n. 2, p. 251-256, 1996. rossef

KIHLSTRAND, M.; STENMAN, B.; NILSON, S.; AXELSSON, O. Water gymnastics reduced the intensity of back low pain in pregnant women. Acta Obstetricia et Gynecologica Scandinavica, v. 78, n. 3, p. 180-185, 1999.

rossef

KLUTHCOVSKY, A. C. G. C.; KLUTHCOVSKY, F. A. O WHOQOL-bref, um instrumento para avaliar qualidade de vida: uma revisão sistemática. Revista de Psiquiatria do Rio Grande do Sul, Porto Alegre, v. 31, n. 3, p. 1-12, 2009. Crossef

KRISTIANSSON, P.; SVÄRDSRDD, K.; SCHOULTZ, B. Back pain during pregnancy: a prospective study. Spine, v. 21, n. 6, p. 702-709, 1996. crossef

MANTLE, M. J.; GREENWOOD, R. M.; CURREY, H. L. F. Backache in pregnancy. Rheumatology and Rehabilitation, v. 16, p. 95-101, 1977. Crossef

MARTINS, R. F.; SILVA, J. L. P. Prevalência de dores na gestação. Revista da Associação Médica Brasileira, São Paulo, v. 51, n. 3, p. 144-147, maio/jun. 2005. Crosšef

MELHADO, S. J. C; SOLER, Z. A. G. A Lombalgia na gravidez: análise entre gestantes no último trimestre da gestação. Femina, v. 32, n. 8, p. 647-652, 2004.

MELZACK, R.; BELANGER, E. L. Pain: correlations with menstrual pain and acute low back pain before and during pregnancy. Pain, v. 36, p. 225-289, 1989. Crossef

MOGREN, I. M; POHJANEN, A. I. Low back pain and pelvic pain during pregnancy: prevalence and risk factors. Spine, v. 30, n. 8, p. 938-991, Apr. 2005. Crossef

MONNERAT, E.; PEREIRA, J. S. Validação e confiabilidade de um questionário para lombalgia. Fitness \& Performance Journal, v. 8, n. 1, p. 45-48, jan./fev. 2009. crossef

NOVAES, F. S.; SHIMO, A. K. K.; LOPES; M. H. B. de M. Lombalgia na gestação. Revista Latinoamericana de Enfermagem, Ribeirão Preto, v. 14, n. 4, p. 620-624, ago. 2006.

OLIVECRONA, M.; RODLING-WAHLSTROM, M.; NAREDI, S.; KOSKINEN, L. O. Prostacylin treatment in severe traumatic brain injury - a microdialysis and outcome study. Journal of Neurotrauma, v. 26, n. 8, p. 1251-1262, 2009. Crossef

ORVIETO, R.; ACHIRON, A.; BEM-RAFAEL, Z.; GELERNTER, I., ACHIRON R. Low back pain of pregnancy. Acta Obstetrícia et Gynecologica Scandinavica, v. 73, n. 3, p. 209-214, Mar. 1994. Crossef 
ÖSTGAARD, H. C.; ANDERSSON, G. B. J. Previus back pain and risk of developing back pain in a future pregnancy. Spine, v. 16, n. 4, p. 432-436, 1991. Crossef

ÖSTGAARD, H. C. Assessment and treatment of low back pain in working pregnant women. Spine, v. 20, n. 1, p. 61-69, 1996. rrossef

PIMENTA, C. A. Fundamentos teóricos da dor e de sua avaliação. In: CARVALHO, M. M. (Org.) Dor: um estudo multidisciplinar. São Paulo: Summus; 1999.

PEDroso, B.; PILATTI, L. A; GUTIERREZ, G. L; PICININ, C. T. Cálculo dos escores e estatística descritiva do WHOQOL-bref através do Microsoft Excel. Revista Brasileira de Qualidade de Vida, Ponta Grossa, v. 2, n. 1, p. 31-36, 2010. Crossef

REZENDE, J.; MONTENEGRO, C. A. B. Obstetrícia Fundamental. 10. ed. Rio de Janeiro: Guanabara Koogan; 2006.

RIBAS, S. I; GUIRRO, E. C. O. Análise da pressão plantar e do equilíbrio postural em diferentes fases da gestação. Brazilian Journal of Physical Therapy, v. 11, n. 5, p. 391-396, set./out. 2007. crossef

RODRIGUES, W. F. G; SILVA, L. R; NASCIMENTO, M. A. L.; PERNAMBUCO, C. S.; GIANI, T. S.; DANTAS, E. H. M. Prevalência de lombalgias e inatividade física: o impacto dos fatores psicossociais em gestantes atendidas pela Estratégia de Saúde da Família. Einstein, São Paulo, v. 9, n. 4, p. 489-493, out./dec. 2011.

SCHORR, S. J.; CHAWLA, A.; DEVIDAS, M.; SULLIVAN, C. A; NAEF, R. W.; MORRISON, J. C. Sleep patterns in pregnancy: a longitudinal study of polysomnography recordings during pregnancy. Journal of Perinatology, v. 18, n. 6, p. 427-430, 1998.

SIHVONEN, T.; HUTTUNEN, M.; MAKKONEM, M.; AIRAKSINEN, O. Functional changes in back muscles activity correlate with pain intensity and prediction of low back pain during pregnancy. Archives of Physical Medicine Rehabilitation, v. 79, p. 1210-1212, 1998. rossef

TIMOSSI, L. S.; PEDROSO, B.; PILATTI, L. A.; FRANCISCO, A. C. Adaptação do modelo de Walton para avaliação da qualidade de vida no trabalho. Revista da Educação Física, Maringá, v. 20, n. 3, p. 394-405, 2009. Crossef

VAN DE POL, G.; DE LEEUW, J. R.; VAN BRUMMEN, H. J.; BRUINSE, H. W.; HEINTZ, A. P.; VAN DER VAART, C. H. The Pregnancy Mobility Index: a mobility scale during and after pregnancy. Acta Obstetricia et Gynecologica Scandinavica, v. 85, n. 7, p. 786-791, 2006. rossef

VALAT, J. P. Factors involved in progression to chronicity of mechanical low back pain. Journal Bone Spine, v. 75, n. 3, p. 193-195, 2005. Crossef

WANG, S. M.; DEZINNO, P.; MARANETS, I.; BERMAN, M. R.; CALDWELL-ANDREWS, A. A.; KAIN, Z. N. Low back pain during pregnancy: prevalence, risk factors, and outcomes. Obstetrics \& Gynecology, v. 104, n. 1, p. 65-70, July 2004. rossef 